\title{
An Older Thrombus Delays Reperfusion after Mechanical Thrombectomy for Ischemic Stroke
}

\author{
Takaya Kitano $^{1,2}$ Yumiko Hori ${ }^{3}$ Shuhei Okazaki ${ }^{1} \quad$ Yuki Shimada $^{4}$ Takanori Iwamoto ${ }^{2}$ \\ Hideaki Kanki ${ }^{1}$ Shintaro Sugiyama ${ }^{1}$ Tsutomu Sasaki ${ }^{1}$ Hajime Nakamura ${ }^{5}$ Naoki Oyama ${ }^{2}$ \\ Taku Hoshi $^{4}$ Goichi Beck ${ }^{1}$ Hiroki Takai ${ }^{6}$ Shunji Matsubara ${ }^{6}$ Hiroya Mizuno $^{7}$ Hirotake Nishimura ${ }^{8}$ \\ Ryo Tamaki $^{9}$ Junichi lida ${ }^{9}$ jiro Iba ${ }^{10}$ Masaaki Uno $^{6}$ Haruhiko Kishima $^{5}$ Hiroaki Fushimi ${ }^{11}$ \\ Satoshi Hattori ${ }^{12,13}$ Shigeo Murayama ${ }^{14,15}$ Eiichi Morii $^{3}$ Manabu Sakaguchi ${ }^{4}$ Yoshiki Yagita ${ }^{2}$

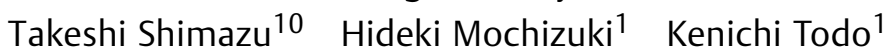

${ }^{1}$ Department of Neurology, Osaka University Graduate School of Medicine, Osaka, Japan

${ }^{2}$ Department of Stroke Medicine, Kawasaki Medical School, Okayama, Japan

${ }^{3}$ Department of Pathology, Osaka University Graduate School of Medicine, Osaka, Japan

${ }^{4}$ Department of Neurology, Osaka General Medical Center, Osaka, Japan

${ }^{5}$ Department of Neurosurgery, Osaka University Graduate School of Medicine, Osaka, Japan

${ }^{6}$ Department of Neurosurgery, Kawasaki Medical School, Okayama, Japan

${ }^{7}$ Department of Cardiovascular Medicine, Osaka University Graduate School of Medicine, Osaka, Japan

8 Department of Pathology, Kawasaki Medical School, Okayama, Japan

${ }^{9}$ Department of Neurosurgery, Osaka General Medical Center, Osaka, Japan

${ }^{10}$ Department of Traumatology and Acute Critical Medicine, Osaka University Graduate School of Medicine, Osaka, Japan

${ }^{11}$ Department of Pathology, Osaka General Medical Center, Osaka, Japan
Address for correspondence Takaya Kitano, MD, Department of Neurology, Graduate School of Medicine, Osaka University, 2-2 Yamadaoka, Suita, Osaka 565-0871, Japan

(e-mail: takayakitano@neurol.med.osaka-u.ac.jp).

Hideki Mochizuki, MD, PhD, Department of Neurology, Graduate School of Medicine, Osaka University, 2-2 Yamadaoka, Suita, Osaka 565-0871, Japan (e-mail: hmochizuki@neurol.med.osaka-u.ac.jp).

${ }^{12}$ Department of Integrated Medicine, Biomedical Statistics, Osaka University Graduate School of Medicine, Osaka, Japan

13 Institute for Open and Transdisciplinary Research Initiatives, Osaka University, Osaka, Japan

${ }^{14}$ Brain Bank for Neurodevelopmental, Neurological and Psychiatric Disorders, United Graduate School of Child Development, Osaka University, Osaka, Japan

${ }^{15}$ Department of Neurology and Neuropathology (the Brain Bank for Aging Research), Tokyo Metropolitan Geriatric Hospital and Institute of Gerontology, Tokyo, Japan

Thromb Haemost 2022;122:415-426.
Abstract
Keywords
- thrombolysis
- embolism
- pathology
- stroke
- macrophage

Background Thrombosis is a dynamic process, and a thrombus undergoes physical and biochemical changes that may alter its response to reperfusion therapy. This study assessed whether thrombus age influenced reperfusion quality and outcomes after mechanical thrombectomy for cerebral embolism.

Methods We retrospectively evaluated 185 stroke patients and thrombi that were collected during mechanical thrombectomy at three stroke centers. Thrombi were pathologically classified as fresh or older based on their granulocytes' nuclear morphology and organization. Thrombus components were quantified, and the extent of NETosis (the process of neutrophil extracellular trap formation) was assessed using the density of citrullinated histone $\mathrm{H} 3$-positive cells. Baseline patient characteristics, thrombus features, endovascular procedures, and functional outcomes were compared according to thrombus age. received

March 2, 2021

accepted after revision

May 22, 2021

published online

June 2, 2021
DOI https://doi.org/

10.1055/a-1522-4507. ISSN 0340-6245.
(C) 2021. The Author(s).

This is an open access article published by Thieme under the terms of the Creative Commons Attribution-NonDerivative-NonCommercial-License, permitting copying and reproduction so long as the original work is given appropriate credit. Contents may not be used for commercial purposes, or adapted, remixed, transformed or built upon. (https://creativecommons.org/ licenses/by-nc-nd/4.0/)

Georg Thieme Verlag KG, Rüdigerstraße 14, 70469 Stuttgart, Germany 
Results Fresh thrombi were acquired from 43 patients, and older thrombi were acquired from 142 patients. Older thrombi had a lower erythrocyte content $(p<0.001)$ and higher extent of NETosis $(p=0.006)$. Restricted mean survival time analysis revealed that older thrombi were associated with longer puncture-to-reperfusion times (difference: 15.6 minutes longer for older thrombi, $p=0.002$ ). This association remained significant even after adjustment for erythrocyte content and the extent of NETosis (adjusted difference: 10.8 minutes, 95\% confidence interval [Cl]: $0.6-21.1$ minutes, $p=0.039$ ). Compared with fresh thrombi, older thrombi required more device passes before reperfusion $(p<0.001)$ and were associated with poorer functional outcomes (adjusted common odds ratio: 0.49; 95\% Cl: 0.24-0.99).

Conclusion An older thrombus delays reperfusion after mechanical thrombectomy for ischemic stroke. Adding therapies targeting thrombus maturation may improve the efficacy of mechanical thrombectomy.

\section{Introduction}

The number of patients with atrial fibrillation is expected to triple the incidence of atrial fibrillation-related cerebral embolism within several decades. ${ }^{1}$ Therefore, the prevention and treatment of cerebral embolism are becoming critical public health issues. Numerous studies have assessed the mechanisms underlying thrombus formation, ${ }^{2}$ and anticoagulation therapy that reduces thrombus formation is recommended for patients with a high risk of embolism. ${ }^{3}$ However, thrombosis is a dynamic process and thrombi undergo changes after their formation, ${ }^{4-6}$ such as fibrin cross-linking that increases elasticity and stiffness over time. ${ }^{7,8}$ Although thrombus age influences the effectiveness of thrombolysis for venous thrombosis, ${ }^{9}$ it remains unclear whether thrombus age affects the treatment and prognosis of cerebral embolism.

The development of mechanical thrombectomy has improved the outcomes after acute large vessel occlusion stroke, ${ }^{10}$ although more than one-half of patients cannot achieve functional independence after this disabling disease. ${ }^{11,12}$ Thus, it is essential to reduce the time from stroke onset to reperfusion in cases of large vessel occlusion stroke, ${ }^{13}$ as a longer puncture-toreperfusion time leads to poorer functional outcomes. ${ }^{14}$ Since thrombus characteristics influence the efficacy of mechanical thrombectomy, ${ }^{15-17}$ we hypothesized that thrombus age might influence the efficacy of mechanical thrombectomy. This study pathologically estimated the age of thrombi that were retrieved during mechanical thrombectomy for cerebral embolism and evaluated whether the thrombus age was associated with puncture-to-reperfusion time, endovascular procedures, and functional outcomes.

\section{Methods}

The data that support the findings of this study are available from the corresponding author upon reasonable request. This study complied with the Declaration of Helsinki and the retrospective study protocol was approved by the appropriate institutional ethics committees. The requirement for written informed consent was waived and patients were allowed to opt out of the research use of their data.

\section{Subjects}

This multicenter retrospective study included patients from three tertiary referral hospitals with comprehensive stroke centers in Japan (Osaka University Hospital, Osaka; Osaka General Medical Center, Osaka; Kawasaki Medical School Hospital, Okayama). We examined 341 consecutive patients who underwent mechanical thrombectomy for acute ischemic stroke between January 2015 and December 2019 and found that thrombus specimens were available for 198 patients. Patients were excluded because of a left ventricular assist device $(n=7)$, atherosclerotic intracranial stenosis $(n=3)$, cerebral artery dissection $(n=2)$, and opting out of research use of data $(n=1)$. Thus, the study included 185 patients with thrombi that were retrieved during mechanical thrombectomy for cerebral embolism (-Fig. 1).

\section{Data Collection}

Data, including age, sex, medical history, prestroke modified Rankin Scale (mRS) score, ${ }^{18}$ stroke subtype, ${ }^{19}$ National Institutes of Health Stroke Scale (NIHSS) score, sites of occluded vessels, laboratory findings, and Alberta Stroke Program Early CT Score (ASPECTS), ${ }^{20}$ which was determined based on computed tomography (CT) and magnetic resonance imaging (MRI) at the admission, were acquired from patients' medical records. If both CT and MRI were performed, MRI findings were prioritized for determining the ASPECTS score. CT-based findings comprised the presence or absence of a hyperdense middle cerebral artery (MCA) sign, ${ }^{21}$ and MRI-based findings were the presence of a susceptibility vessel sign on $\mathrm{T}^{*}$ images. ${ }^{22}$ Functional outcome scores based on the mRS were collected at 3 months after stroke onset.

\section{Endovascular Procedures}

We reviewed the use of intravenous recombinant tissue-type plasminogen activator (rt-PA) and the technical details of each endovascular procedure. The strategies for treating acute ischemic stroke were decided by the attending physicians. The total number of thrombectomy device passes, which were attempted before angiographic reperfusion or at the end of the procedure, was assessed for each patient. Endovascular procedures, such as balloon angioplasty or stenting, were not counted if they did not 


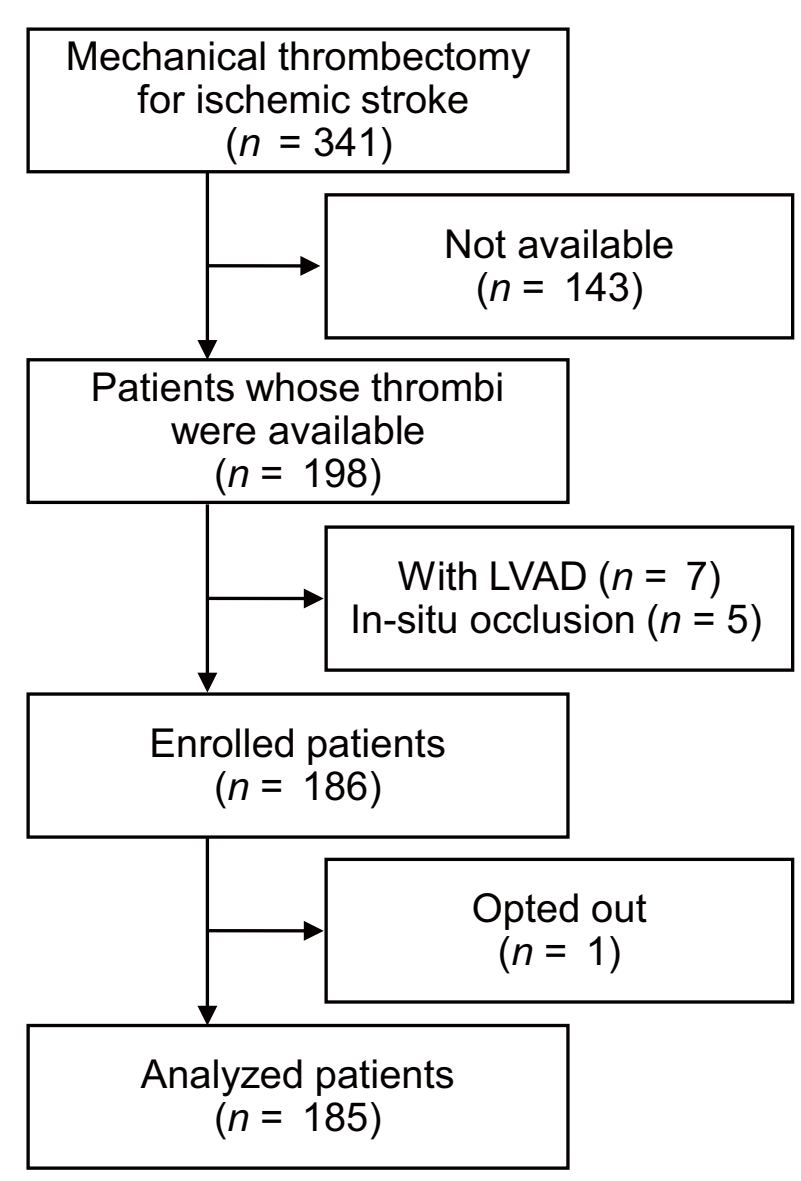

Fig. 1 Study population. LVAD, left ventricular assist device.

attempt to retrieve a thrombus, and the use of an aspiration catheter as a distal access device was not counted. Based on the device that was used, we classified the endovascular procedures as "catheter aspiration," "stent retriever," and "combined." Successful angiographic reperfusion was identified based on grade $2 \mathrm{~b}$ or greater using the expanded Thrombolysis in Cerebral Infarction (eTICI) system. ${ }^{23}$

\section{Sample Preparation and Immunohistochemical Staining}

Retrieved thrombi were immediately fixed in $10 \%$ neutral buffered formalin, embedded in paraffin, and cut into $4-\mu \mathrm{m}-$ thick sections. All samples were collected and analyzed at the Osaka University laboratory.

Serial sections were stained using hematoxylin and eosin (H\&E) and phosphotungstic acid-hematoxylin. Immunohistochemical staining was performed using a Roche Ventana BenchMark GX autostainer (Ventana Medical Systems, Tucson, Arizona, United States) according to the manufacturer's instructions. The primary antibodies targeted CD42b (sc-80728, 1:200; Santa Cruz Biotechnology, Dallas, Texas, United States), CD163 (NCL-L-CD163, 1:100; Leica Biosystems, Wetzlar, Germany), $\alpha-$ smooth muscle actin ( $\alpha$-SMA; M0851, 1:800; Agilent Technologies, Santa Clara, California, United States), and citrullinated histone H3 (H3Cit) (ab5103, 1:3200; Abcam, Cambridge, United Kingdom). Stained slides were examined using a Nikon Eclipse 80i microscope (Nikon, Tokyo, Japan).
Quantification of Thrombus Components and NETosis Whole microscopic digital images of the slides were captured using the NanoZoomer Digital Pathology System (Hamamatsu Photonics, Hamamatsu, Japan). Examples of quantification are shown in -Supplementary Fig. S1 (available in the online version). The thrombus size was evaluated based on the cross-sectional area, and each component of the specimen was semi-automatically quantified using the entire specimen with the Fiji-ImageJ software package. ${ }^{24}$ Compared with the area of the entire specimen, the proportional areas were evaluated for red blood cells (RBCs) using H\&E staining, fibrin using phosphotungstic acid-hematoxylin staining, and platelets using immunohistochemical staining for CD42b. The densities of white blood cells and CD163positive cells were evaluated based on H\&E staining and immunohistochemical staining.

NETosis is the process of extracellular trap formation by thread-like structures of decondensed DNA that are decorated with proteins from cytoplasmic granules. ${ }^{25}$ The extent of NETosis in thrombi was evaluated using the density of H3Citpositive cells in all patients because H3Cit is a marker for immune cells that are about to release extracellular traps. ${ }^{26}$ The density of H3Cit-positive cells was assessed based on immunohistochemical staining as described above.

\section{Thrombus Age}

Thrombus age was estimated via two strategies. First, the retrieved thrombus was pathologically classified using the accepted definitions ${ }^{27}$ : a fresh thrombus ( $<1$ day) that is composed of layered patterns of platelets, fibrin, erythrocytes, and intact granulocytes; a lytic thrombus (1-5 days) that is characterized by areas of colliquation necrosis and granulocyte karyorrhexis; and an organized thrombus ( $>5$ days) that exhibits spindle-shaped cell ingrowth and positivity for anti- $\alpha$-SMA with or without connective tissue deposition and capillary vessel ingrowth. As a thrombus may present mixed fresh, lytic, or organization features, we judged a thrombus as fresh only when intact granulocytes were dominantly observed thoroughly ( $>80 \%$ ) over a section, while we judged a thrombus as lytic when the areas of colliquation necrosis and granulocyte karyorrhexis were $>20 \%$. When a section showed positivity for $\alpha$-SMA staining even if it was a little part, the thrombus was judged as organized. For the present study, we classified lytic and organized thrombi as "older" thrombi. The anonymized specimens were pathologically evaluated by an experienced interventionalist (T.K.) with no attached clinical information. Interobserver agreement regarding thrombus age estimation was measured using $\mathrm{k}$-statistics and weighted $\mathrm{k}$-statistics, based on independent assessments of all samples that were performed by an experienced pathologist (Y.H.).

The second strategy, which was used to enhance the robustness of the findings, evaluated the density of CD163positive cells to determine thrombus age. CD163 is expressed exclusively on circulating monocytes and tissue macrophage subpopulations, ${ }^{28,29}$ and CD163-positive cell density has been reported to positively correlate with thrombus age in patients with deep venous thrombosis. ${ }^{30} \mathrm{~A}$ rabbit model of jugular 
venous thrombus has also indicated that the number of macrophages in the thrombus increases over time. ${ }^{31}$ However, it is important to note that CD 163-positive cell density increases during the first few days after thrombosis formation and then decreases slowly. ${ }^{32}$ Therefore, we divided the thrombi according to tertiles of CD163-positive cell density based on the assumption that thrombi in the lowest tertile were younger than those in the middle and highest tertiles.

\section{Statistical Analysis}

Baseline characteristics, endovascular procedure details, puncture-to-reperfusion times, and functional outcomes were compared between patients with fresh and older thrombi. The cumulative rate of successful reperfusion according to thrombus age was evaluated using the Kaplan-Meier method and the generalized Wilcoxon test. We did not censor the observation at the end of the procedure. We also compared the puncture-to-reperfusion times between the lowest CD163-positive tertile and the middle/highest tertiles. The puncture-to-reperfusion time according to thrombus age was analyzed using restricted mean survival time (RMST) analysis. ${ }^{33,34}$ In this study, the RMST reflected the average puncture-to-reperfusion time within a specified truncation time and was evaluated as the area under the survival curve. ${ }^{34}$ The truncation time was set to 120 minutes because we considered that puncture-toreperfusion time $>120$ minutes is less clinically important. The RMST difference was calculated according to thrombus age and after adjusting for confounders using the RMST regression analysis with the pseudo-value technique. The adjusted confounders were age, sex, occluded vessels, NIHSS score, rt-PA administration, thrombus size, RBC proportion, and density of H3Cit-positive cells in the thrombus. The occluded vessels were classified as extracranial vessels (common carotid artery and extracranial internal carotid artery [ICA]), anterior circulation (intracranial ICA and MCA), and posterior circulation (vertebral artery, basilar artery, and posterior cerebral artery). Subgroup analyses were performed using the RMST regression analysis to examine heterogeneity in the effects of thrombus age. The study population was subdivided according to age ( $<80$ years or $\geq 80$ years), sex, presence or absence of atrial fibrillation, NIHSS score ( $<18$ or $\geq 18$ ), occluded vessel (intracranial ICA, the horizontal segment of the MCA, or distal MCA), and rt-PA administration. To quantitatively evaluate the heterogeneity, interactions were tested using a multiplicative interaction term (thrombus age * variable) in the models with dichotomization of continuous variables.

Sensitivity analyses were also performed to test the stability of the findings. First, we applied a three-category classification system (fresh, lytic, and organized) instead of the two-category system (fresh and older). Second, we only analyzed patients who experienced complete or nearcomplete reperfusion because the retrieved specimen may not represent the entire thrombus in patients with partial reperfusion. Third, we modified the truncation time in the RMST-based analyses. Finally, we constructed a Cox proportional hazard model.
The functional outcome was examined based on the shift in the distribution of the mRS scores at 90 days between the thrombus age groups, with scores of 5 (bed-bound with severe disability) and 6 (death) combined. Adjusted common odds ratios were calculated using a multivariable ordinal logistic regression (proportional odds regression model). The adjusted variables were age, sex, occluded vessels, NIHSS score, rt-PA administration, onset-to-puncture time, thrombus size, RBC proportion, and H3Cit-positive cells density in the thrombus. As a sensitivity analysis, we imputed the missing outcomes with multiple imputations $(n=20$ imputation sets). Predictors in the imputation model included age, sex, NIHSS, occluded vessels, rt-PA administration, onset-topuncture time, procedure time, and reperfusion grade (eTICI). The fully conditional specification method was used for generating imputed samples, and Rubin's rule was used for estimating standard errors.

The number of device passes, before achieving successful reperfusion or at the end of the procedure, and the proportion of first-pass reperfusion were compared between patients with fresh and older thrombi. The proportion of first-pass reperfusion was also compared according to the thrombus age group and endovascular procedure.

Continuous variables are reported as the median and interquartile range (IQR), while categorical variables are reported as number and percentage. Continuous variables were compared using the Wilcoxon rank-sum test, and categorical variables were compared using Fisher's exact test unless otherwise specified. Statistical significance was established at $p$-values $<0.05$. All analyses were performed using SAS university edition (SAS 9.4, SAS Institute Inc., Cary, North Carolina, United States).

\section{Results}

\section{Baseline Characteristics}

We enrolled a total of 185 patients with thrombi that were retrieved during mechanical thrombectomy for cerebral embolism. The thrombi were classified as fresh in 43 patients (23\%), lytic in 131 patients (71\%), and organized in 11 patients (6\%). The weighted $\mathrm{K}$-statistic was 0.91 and the $\mathrm{K}-$ statistic was 0.53 when lytic/organized thrombi were classified as "older" thrombi. Typical microscopic images of each thrombus category are shown in - Fig. 2 . The pathologically defined thrombus age was associated with CD163-positive cell density, and older thrombi had a greater density of CD163-positive cells compared with fresh thrombi (median IQR: $185 / \mathrm{mm}^{2}$ [88-234/mm²] vs. $328 / \mathrm{mm}^{2}$ [194-446/mm ${ }^{2}$, $p<0.001)$. The patients' baseline characteristics according to pathologically defined thrombus age are shown in - Table $\mathbf{1}$. Relative to fresh thrombi, older thrombi were associated with a higher prevalence of diabetes ( 7 vs. $21 \%, p=0.04$ ) and a higher median concentration of C-reactive protein ( $0.13 \mathrm{vs}$. $0.21 \mathrm{mg} / \mathrm{dL}, p=0.015$ ). In addition, older thrombi were marginally associated with a higher concentration of brain natriuretic peptide (BNP) (100 vs. $202 \mathrm{pg} / \mathrm{mL}, p=0.052)$. There were no significant intergroup differences in stroke subtypes, sites of occluded vessels, onset-to-puncture times, 

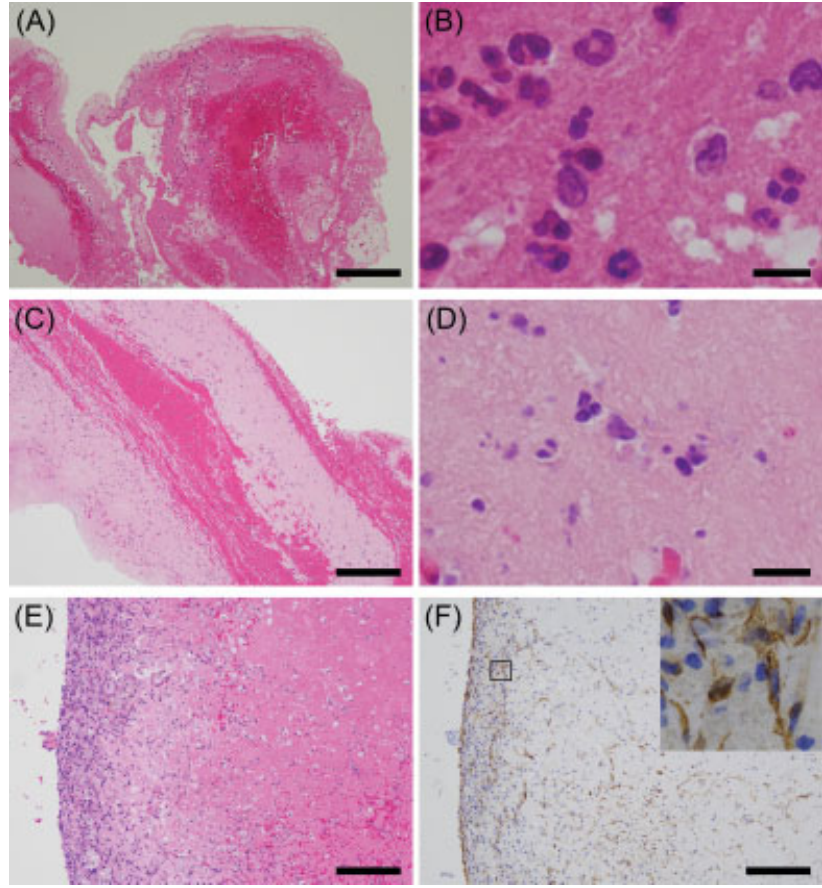

Fig. 2 Representative images of the retrieved thrombi according to age group. (A-E) Hematoxylin and eosin staining. (A) Low-power view of a fresh thrombus and (B) a high-power view of panel (A). Granulocytes with intact nuclei are visible. (C) Low-power view of a lytic thrombus and (D) a high-power view of panel (C). Granulocytes with karyorrhexis and nuclear dust are visible. (E) Low-power view of an organized thrombus and (F) immunohistochemical staining showing $\alpha$-SMA-positive spindle cells mainly at the margin of the thrombus. The boxed area is magnified. (A, C, E, F) Bar $=200 \mu \mathrm{m}$. (B, D) Bar $=10$ $\mu \mathrm{m}$. $\alpha$-SMA, $\alpha$-smooth muscle actin.

the prevalence of the hyperdense MCA sign, and prevalence of the susceptibility vessel sign. The patients' baseline characteristics according to CD163-positive tertiles are presented in -Supplementary Table $\mathbf{S 1}$ (available in the online version).

\section{Thrombus Age and Pathological Features}

We compared thrombus components according to age (-Table 2) and revealed that older thrombi had a lower proportion of RBC content, higher white blood cell density, and higher platelet content. The extent of NETosis was evaluated by immunohistochemically staining the thrombi for H3Cit, which identifies immune cells that are primed to release extracellular traps. Compared with fresh thrombi, older thrombi had a significantly higher median density of H3Cit-positive cells $\left(236 / \mathrm{mm}^{2}\right.$ [IQR: $118-368 / \mathrm{mm}^{2}$ ] vs. $355 / \mathrm{mm}^{2}$ [IQR: $171-741 / \mathrm{mm}^{2}$ ], $\left.p=0.006\right)$.

\section{Thrombus Age and Time to Reperfusion}

Cumulative rates of successful reperfusion after puncture according to thrombus age are shown in - Fig. 3. Older thrombi were associated with a significantly longer time to reperfusion ( $p=0.004$ ). The RMSTs for successful reperfusion were 55.9 minutes for fresh thrombi and 71.5 minutes for older thrombi (average of 15.6 minutes longer for older thrombi, $p=0.002$ ). Similarly, relative to the lowest
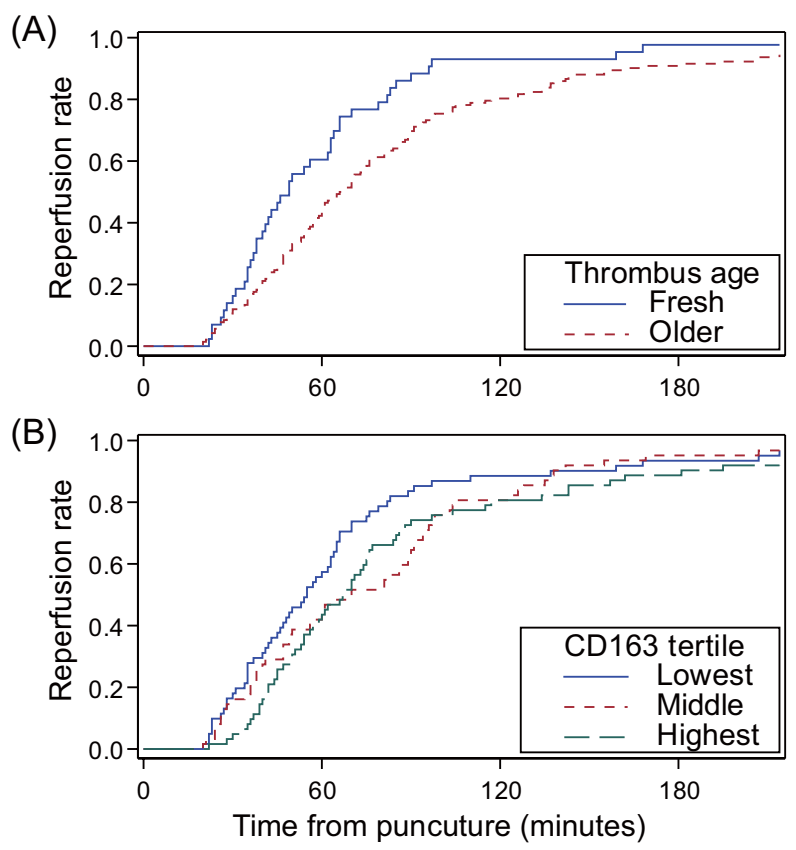

Fig. 3 Cumulative rate of reperfusion after puncture. (A) According to pathologically defined thrombus age, patients with older thrombi had longer times to reperfusion ( $p=0.004$, generalized Wilcoxon test). The restricted mean survival time (RMST) for successful reperfusion was 55.9 minutes in patients with fresh thrombi and

71.5 minutes in patients with older thrombi $(p=0.002)$. (B) According to the density of CD163-positive cells in the thrombi, and relative to thrombi in the lower tertile, longer times to reperfusion were observed for thrombi in the middle tertile $(p=0.075)$ and in the highest tertile $(p=0.019)$. The RMST for successful reperfusion was 59.6 minutes for thrombi in the lowest tertile, 71.6 minutes for the middle tertile $(p=0.036)$, and 72.3 minutes for the highest tertile $(p=0.019)$. The truncation time was 120 minutes.

CD163-positive tertile, longer times to reperfusion were required for thrombi in the middle tertile $(p=0.075)$ and the highest tertile $(p=0.019)$ (-Fig. 3 ). The RMSTs for successful reperfusion were 59.6 minutes for the lowest CD163-positive tertile, 71.6 minutes for the middle tertile ( +12.0 minutes, $p=0.036$ ), and 72.3 minutes for the highest tertile $(+12.7$ minutes, $p=0.019)$.

A multivariate analysis revealed that, relative to fresh thrombi, older thrombi were associated with longer puncture-to-reperfusion times even after adjustment for possible confounding factors, including RBC content and the extent of NETosis (adjusted RMST difference: 10.8 minutes, 95\% confidence interval [CI]: 0.6-21.1 minutes; $p=0.039$ ) ( - Table 3). Extracranial vessel occlusion and lower RBC content were also associated with longer puncture-to-reperfusion times. Similarly, relative to the lowest CD163-positive tertile, longer puncture-to-reperfusion times were observed for thrombi in the middle tertile (adjusted RMST difference: 13.0 minutes, 95\% CI: 2.5-23.5 minutes; $p=0.015$ ), but not for those in the highest tertile (adjusted RMST difference: 6.8 minutes, 95\% CI: -3.4 to 17.0 minutes; $p=0.194$ ).

In subgroup analyses, there was no significant heterogeneity in the effect of thrombus age (-Fig. 4). 
Table 1 Baseline patient characteristics

\begin{tabular}{|c|c|c|c|c|}
\hline & $\begin{array}{l}\text { Fresh thrombi } \\
(n=43)\end{array}$ & $\begin{array}{l}\text { Older thrombi } \\
(n=142)\end{array}$ & $p$-Value & Missing \\
\hline Age, y & $77(67-84)$ & $80(70-84)$ & 0.474 & 0 \\
\hline Male sex & $25(58 \%)$ & $75(53 \%)$ & 0.602 & 0 \\
\hline Hypertension & $32(74 \%)$ & $85(60 \%)$ & 0.104 & 0 \\
\hline Diabetes & $3(7 \%)$ & $30(21 \%)$ & 0.040 & 0 \\
\hline Dyslipidemia & $16(37 \%)$ & $46(32 \%)$ & 0.583 & 0 \\
\hline Atrial fibrillation & $28(65 \%)$ & $101(71 \%)$ & 0.455 & 0 \\
\hline Antiplatelet use & $10(23 \%)$ & $35(25 \%)$ & 1.000 & 0 \\
\hline Anticoagulant use & $10(23 \%)$ & $30(21 \%)$ & 0.833 & 0 \\
\hline Modified Rankin Scale & $0(0-2)$ & $0(0-2)$ & 0.743 & 0 \\
\hline NIHSS score & $19(13-26)$ & $17(12-24)$ & 0.313 & 0 \\
\hline Stroke subtype & & & 0.820 & 0 \\
\hline Cardioembolic & $31(72)$ & $105(74)$ & & \\
\hline Large artery atherosclerosis & $2(5)$ & $9(6)$ & & \\
\hline Other $^{a}$ & $10(23)$ & $28(20)$ & & \\
\hline Leukocyte count, / $\mu \mathrm{L}$ & $7,260(6,280-10,260)$ & $7,505(5,970-9,440)$ & 0.775 & 0 \\
\hline Platelet count, $\times 10^{3} / \mu \mathrm{L}$ & $200(171-252)$ & $193(159-235)$ & 0.327 & 0 \\
\hline C-reactive protein, $\mathrm{mg} / \mathrm{dL}$ & $0.13(0.06-0.30)$ & $0.21(0.1-0.84)$ & 0.015 & 0 \\
\hline D-dimer, $\mu \mathrm{g} / \mathrm{mL}$ & $1.4(0.70-3.49)$ & $1.83(1-4.61)$ & 0.234 & 5 \\
\hline Brain natriuretic peptide, $\mathrm{pg} / \mathrm{mL}$ & $100(53-311)$ & $202(80-368)$ & 0.052 & 22 \\
\hline ASPECTS & $7(5-9)$ & $9(6-10)$ & 0.071 & $19^{b}$ \\
\hline Occluded vessels & & & 0.151 & 0 \\
\hline Extracranial vessel & $4(9)$ & $14(10)$ & & \\
\hline Intracranial ICA & $9(21)$ & $33(23)$ & & \\
\hline M1 & $18(42)$ & $53(37)$ & & \\
\hline $\mathrm{M} 2$ & $4(9)$ & $31(22)$ & & \\
\hline Posterior circulation & $8(19)$ & $11(8)$ & & \\
\hline Tandem lesions & $6(14 \%)$ & $12(9 \%)$ & 0.376 & 0 \\
\hline Hyperdense MCA sign & $6(46 \%)$ & $31(60 \%)$ & 0.533 & 120 \\
\hline Susceptibility vessel sign & $19(57 \%)$ & $62(65 \%)$ & 0.530 & 57 \\
\hline rtPA administration & $10(23 \%)$ & $52(37 \%)$ & 0.140 & 0 \\
\hline Onset-to-puncture time, min & $237(147-365)$ & $220(141-366)$ & 0.516 & 0 \\
\hline
\end{tabular}

Abbreviations: ASPECTS, Alberta Stroke Program Early CT Score; ICA, internal carotid artery; MCA, middle cerebral artery; NIHSS, National Institutes of Health Stroke Scale; rtPA, recombinant tissue-type plasminogen activator.

Note: Data are presented as median (interquartile range) or number (percentage).

${ }^{\mathrm{a}}$ Other determined etiology and undetermined etiology.

bPatients with posterior circulation occlusion.

\section{Sensitivity Analysis}

Thrombus age was reclassified using a three-category system (fresh, lytic, and organized), which revealed that, relative to fresh thrombi, longer times to reperfusion were observed for lytic thrombi $(p=0.011)$ and organized thrombi $(p=0.008)$ (-Supplementary Fig. S2, available in the online version). The RMSTs for successful reperfusion were 70.3 minutes for lytic thrombi ( $p=0.004$ [vs. fresh thrombi]) and 85.8 minutes for organized thrombi ( $p=0.001$ [vs. fresh thrombi]). We also restricted the analyses to patients who achieved complete or near-complete reperfusion (-Supplementary Fig. S3, available in the online version), which revealed RMSTs of 54.9 minutes for fresh thrombi and 68.6 minutes for older thrombi $(p=0.017)$. A third sensitivity analysis involved changing the truncation time to $90,150,180$, or 214 minutes in the RMST analyses, although these revealed consistent results ( - Supplementary Table S2, available in the online version). Finally, we constructed a proportional hazard model, instead of the RMST-based analysis, which showed that older thrombi were marginally associated with longer puncture-to-reperfusion times (hazard ratio: 0.69, 
Table 2 Thrombus components according to age

\begin{tabular}{|l|l|l|l|}
\hline & Fresh thrombi & Older thrombi & $p$-Value \\
\hline Area, $\mathrm{mm}^{2}$ & $15.0(6.8-26.4)$ & $10.9(6.5-19.3)$ & 0.290 \\
\hline Red blood cells, \% & $53(39-65)$ & $35(23-50)$ & $<0.001$ \\
\hline White blood cell density, $/ \mathrm{mm}^{2}$ & $1,828(1,067-2,103)$ & $2,204(1,494-3,284)$ & 0.011 \\
\hline Platelets, \% & $17(12-27)$ & $24(17-32)$ & 0.025 \\
\hline Fibrin, \% & $28(20-44)$ & $35(23-45)$ & 0.119 \\
\hline H3Cit-positive cell density, $/ \mathrm{mm}^{2}$ & $236(118-368)$ & $355(171-741)$ & 0.006 \\
\hline
\end{tabular}

Abbreviation: H3Cit, citrullinated histone $\mathrm{H} 3$.

Note: Data are presented as median (interquartile range).

95\% CI: $0.47-1.00 ; p=0.053$ ) (-Supplementary Table S3, available in the online version).

\section{Thrombus Age and Endovascular Procedures}

To elucidate why older thrombi were associated with prolonged puncture-to-reperfusion times, we compared endovascular procedures according to thrombus age. Relative to fresh thrombi, older thrombi required significantly more device passes (median: 1 pass vs. 2 passes, $p<0.001$ ) (-Fig. 5). Furthermore, older thrombi had a lower proportion of successful reperfusion after the first pass ( 72 vs. $45 \%$, $p=0.003$ ), and the results according to the first endovascular procedure are shown in -Fig. 5. There was no significant heterogeneity in the effect of thrombus age on the proportion of first-pass reperfusion according to the endovascular procedure $(p$ for interaction $=0.831)$.

\section{Thrombus Age and Functional Outcomes}

Functional outcomes were assessed based on the 3-month mRS scores (-Fig. 6), although six patients were lost to follow-up and excluded from this analysis. Older thrombi were marginally associated with poorer functional outcomes (common odds ratio: $0.59,95 \% \mathrm{CI}: 0.31-1.10 ; p=0.096$ ). After adjustment for covariates, older thrombi were significantly associated with poorer functional outcomes (adjusted common odds ratio: $0.49,95 \% \mathrm{CI}: 0.24-0.99 ; p=0.047)$. The pooled effect on functional outcomes was similar following multiple imputation (adjusted common odds ratio: $0.71,95 \%$ CI: $0.49-1.01 ; p=0.057$ ).

\section{Discussion}

This study pathologically estimated thrombus age and evaluated whether the thrombus age was associated with the outcomes of mechanical thrombectomy for cerebral embolism. The main findings are summarized in the Visual Summary (-Fig. 7). Thrombi in cerebral embolism were mainly fresh $(23 \%)$ or lytic (71\%), while organized thrombi were uncommon (6\%). Relative to fresh thrombi, older thrombi required more device passes and had longer puncture-to-reperfusion times even after adjusted for possible confounding factors, and the deterioration of the quality of reperfusion resulted in poorer functional outcomes.

Table 3 Adjusted RMST difference within 120 minutes after puncture

\begin{tabular}{|c|c|c|c|}
\hline & Adjusted RMST difference (min) & $95 \% \mathrm{Cl}$ & $p$-Value \\
\hline Age, per 10-year increase & 1.0 & $-2.2,4.2$ & 0.538 \\
\hline Male sex & -5.2 & $-13.3,2.8$ & 0.200 \\
\hline NIHSS score, per 1-point increase & -0.4 & $-1.0,0.2$ & 0.155 \\
\hline \multicolumn{4}{|l|}{ Occluded vessels ${ }^{a}$} \\
\hline Extracranial vessels & 49.1 & $37.7,60.4$ & $<0.001$ \\
\hline Posterior circulation & 11.0 & $-5.1,27.0$ & 0.181 \\
\hline rt-PA administration & -2.8 & $-11.2,5.6$ & 0.515 \\
\hline Older thrombus & 10.8 & $0.6,21.1$ & 0.039 \\
\hline RBC content, per $10 \%$ increase & -2.8 & $-5.2,-0.4$ & 0.022 \\
\hline H3Cit-positive cells density, per $100 / \mathrm{mm}^{2}$ increase & 0.5 & $-0.5,1.6$ & 0.329 \\
\hline Thrombus size, per $10-\mathrm{mm}^{2}$ increase & -0.5 & $-1.5,0.5$ & 0.307 \\
\hline
\end{tabular}

Abbreviations: $\mathrm{Cl}$, confidence interval; H3Cit, citrullinated histone H3; NIHSS, National Institutes of Health Stroke Scale; RBC, red blood cell; RMST, restricted mean survival time; rt-PA, recombinant tissue-type plasminogen activator.

${ }^{a}$ Versus the intracranial anterior circulation. 


\begin{tabular}{|c|c|c|c|c|c|}
\hline \multirow{2}{*}{$\begin{array}{c}\text { Subgroups } \\
\text { Overall }\end{array}$} & \multirow{2}{*}{$\begin{array}{l}\text { No. of } \\
\text { patients } \\
\text { Fresh }\end{array}$} & \multirow{2}{*}{$\begin{array}{l}\begin{array}{l}\text { No. of } \\
\text { patients } \\
\text { Older }\end{array} \\
142\end{array}$} & $\begin{array}{l}\text { Difference } \\
\text { in RMST }\end{array}$ & Estimates $(95 \% \mathrm{Cl})$ & \multirow[t]{2}{*}{ Interaction } \\
\hline & & & & $10.8(0.6-21.1)$ & \\
\hline Age $<80$ y & 25 & 69 & & $17.7(3.7-31.6)$ & \multirow{2}{*}{0.138} \\
\hline Age $\geq 80$ y & 18 & 73 & & $1.1(-15.2-17.5)$ & \\
\hline \multirow{2}{*}{$\begin{array}{l}\text { Female } \\
\text { Male }\end{array}$} & 18 & 67 & $H$ & $12.2(-2.8-27.2)$ & \multirow{2}{*}{0.533} \\
\hline & 25 & 75 & $\longmapsto$ & $8.7(-5.8-23.1)$ & \\
\hline \multirow{2}{*}{$\begin{array}{c}\text { No AF } \\
\text { AF }\end{array}$} & 15 & 41 & $\mapsto$ & $10.0(-5.0-25.0)$ & \multirow{2}{*}{0.916} \\
\hline & 28 & 101 & $\mapsto$ & $9.1(-3.8-22.0)$ & \\
\hline \multirow{2}{*}{$\begin{array}{l}\text { NIHSS }<18 \\
\text { NIHSS } \geq 18\end{array}$} & 18 & 73 & - & $3.7(-10.8-18.3)$ & \multirow{2}{*}{0.180} \\
\hline & 25 & 69 & & $-18.5(4.6-32.4)$ & \\
\hline \multirow{3}{*}{$\begin{array}{l}\text { ICA } \\
\text { M1 } \\
\text { M2 }\end{array}$} & 9 & 33 & - & $-1.2(-19.5-17.1)$ & \multirow{3}{*}{0.282} \\
\hline & 18 & 53 & $H$ & $11.4(-2.6-25.5)$ & \\
\hline & 4 & 31 & & $4.7(-20.0-29.4)$ & \\
\hline \multirow{3}{*}{$\begin{array}{c}\text { No rtPA } \\
\text { rtPA }\end{array}$} & 33 & 90 & & $13.1(0.1-26.2)$ & \multirow{2}{*}{0.383} \\
\hline & 10 & 52 & $\rightarrow$ & $3.6(-12.6-20.0)$ & \\
\hline & & -30 & 0 & (minutes) & \\
\hline
\end{tabular}

Fig. 4 Effect of thrombus age on RMST in the subgroups. The adjusted RMST differences and their $95 \% \mathrm{Cls}$ are shown for patients with fresh and older thrombi. There was no significant interaction. The truncation time was 120 minutes. AF, atrial fibrillation; $\mathrm{Cl}$, confidence interval; ICA, internal carotid artery; M1, the horizontal segment of the middle cerebral artery; M2, the insular segment of the middle cerebral artery; NIHSS, National Institutes of Health Stroke Scale; RMST, restricted mean survival time; rtPA, recombinant tissue-type plasminogen activator.

\section{Thrombus Characteristics and Resistance to Mechanical Thrombectomy}

Thrombus characteristics influence the efficacy of mechanical thrombectomy. ${ }^{15-17}$ The lower RBC content (or higher platelet/fibrin content) in thrombi is associated with longer puncture-to-reperfusion times. ${ }^{35,36}$ An experimental model also indicated that RBC content influences mechanical thrombectomy as arteries occluded by fibrin-rich clot analogs required significantly longer recanalization times than arteries occluded by RBC-rich clot. ${ }^{37}$ In addition, higher levels of NETosis in thrombi may delay recanalization in patients with cerebral embolism. ${ }^{38}$ NETosis is the process of extracellular trap formation by thread-like structures of decondensed DNA that are decorated with proteins from cytoplasmic granules. ${ }^{25}$ NETosis renders thrombi resistant to mechanical and enzymatic destruction, ${ }^{39}$ while DNAse 1 accelerated rt-PA-induced thrombolysis in a study of thrombi retrieved from stroke patients. ${ }^{40}$

This study demonstrated that thrombus age was associated with the quality of reperfusion and functional outcomes. As previously reported, ${ }^{30,41}$ older thrombi had a lower erythrocyte content and higher extent of NETosis; however, the effect of thrombus age on mechanical thrombectomy remained significant even after adjustment for these factors. This significance of thrombus age in this context may be reasonable because a thrombus changes dynamically after its formation. ${ }^{4-6}$ For instance, fibrin cross-linking increases elasticity and stiffness over time ${ }^{7,8}$ and thrombus stiffness increases as the thrombus ages. ${ }^{42,43}$ Stiff thrombus would be difficult to manage during 

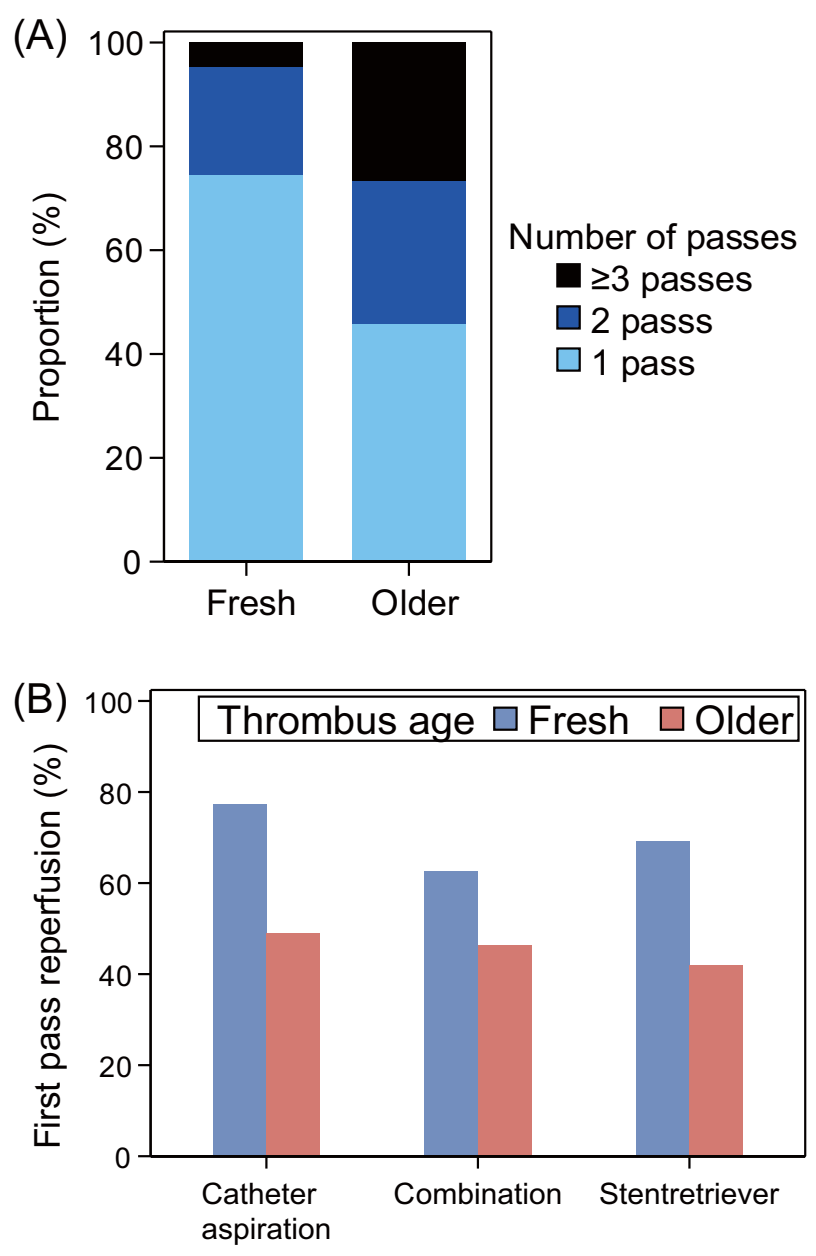

Fig. 5 Association between thrombus age and number of device passes. (A) The number of device passes needed to achieve reperfusion was higher in patients with older thrombi $(p<0.001)$. (B) The proportions of patients who achieved first-pass reperfusion are shown according to the first device used and thrombus age.

mechanical thrombectomy as it may be less likely to be aspirated or entangled in the stent struts. Our study highlighted the need to elucidate the pathophysiological role of thrombus aging in embolisms.

\section{Using RMST-Based Analysis of Time to Reperfusion}

We performed an RMST-based analysis of the puncture-toreperfusion time, although a Cox proportional hazard model is often used for survival analyses. This is because the Cox model relies on the assumption of proportional hazards (i.e., the ratio of the hazard curve is constant over time). However, this assumption is implausible when analyzing puncture-toreperfusion time for mechanical thrombectomy, as successful reperfusion is rarely observed during the first 10 minutes (because of the time needed to guide the large catheter to the carotid artery), and thereafter, the rate of reperfusion rapidly increases at first and then reaches a plateau. In contrast, the RMST examines the average time-to-event over a restricted follow-up period and is generally limited by the need to specify a truncation time. ${ }^{33}$ However, this limitation may be less important when analyzing time to reperfusion for

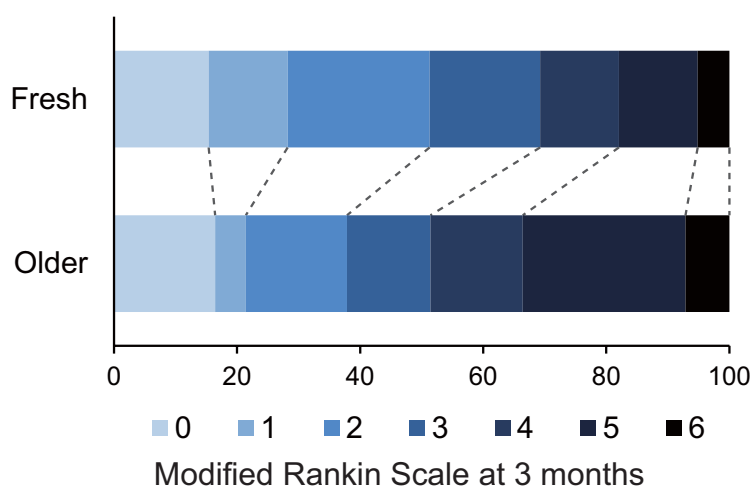

Fig. 6 Distribution of the modified Rankin Scale score at 3 months. The modified Rankin Scale scores at 3 months are shown. The adjusted common odds ratio was 0.49 (95\% confidence interval: $0.49-0.99$; $p=0.047)$.

mechanical thrombectomy, as the benefit of reperfusion decreases over time and the point of clinical interest is the change in the reperfusion rate during the period relatively early after the puncture. Thus, we initially set the truncation time to 120 minutes, although sensitivity analyses with different truncation times revealed relatively consistent results. Consequently, the results from the RMST-based analysis and a proportional hazard ratio-based analysis were generally consistent.

\section{Factors That Affect Thrombus Age in Cerebral Embolism}

In cases of cerebral embolism, thrombi are mainly cardiogenic and related to atrial fibrillation. Thus, a plausible determinant of thrombus age is the interval from thrombus formation to embolization, with older thrombi spending relatively longer times in the left atrium or appendage. It is not possible that the thrombi aged in the embolized artery, as the onset-to-puncture time was not associated with thrombus age. In our cohort, patients with older thrombi (based on pathological assessment or CD163-positive cell density) tended to have higher concentrations of BNP. Thus, thrombi may remain in the heart for longer periods when cardiac function is low, which would result in the retrieval of older thrombi.

Thrombus age might also be related to a patient's condition, as we found that patients with older thrombi were more likely to have diabetes and higher concentrations of C-reactive protein. The presence of diabetes primes neutrophils to undergo NETosis ${ }^{44}$ and inflammation in thrombi attracts monocyte-derived macrophages that cross-link fibrin by secreting factor XIIIa. ${ }^{4,45}$ Therefore, diabetes and proinflammatory conditions may accelerate thrombus maturation. Further studies are needed to clarify the determinants of thrombus age in embolism.

\section{Estimating the Age of the Retrieved Thrombi}

There are few studies regarding thrombus age in cerebral embolism. Niesten et al evaluated 22 thrombi that were retrieved from cerebral embolism cases between 2010 and 


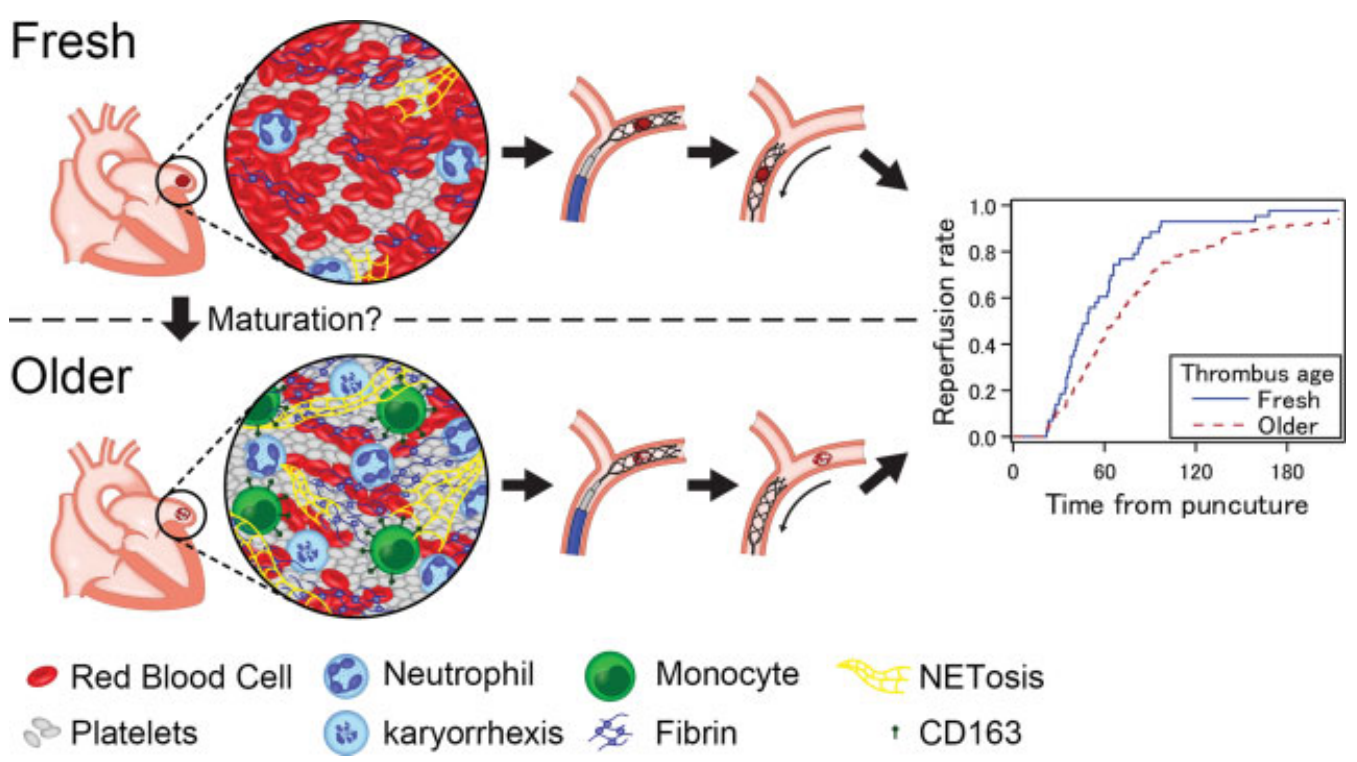

Fig. 7 Visual Summary. We pathologically estimated the ages of thrombi that were retrieved from patients with cerebral embolism (mainly cardiogenic) via mechanical thrombectomy. Fresh thrombi and older thrombi were identified. The density of CD163-positive cells (monocytes or macrophages) was higher in older thrombi. Relative to fresh thrombi, older thrombi required more device passes and had a longer puncture-toreperfusion time; these findings were independent of erythrocyte content and the extent of NETosis. The deterioration of the quality of reperfusion resulted in poorer functional outcomes.

2013 and reported that 16 thrombi were fresh, 4 thrombi were lytic, and 2 thrombi were organized. ${ }^{46}$ Laridan et al also reported that 32 of 68 thrombi were fresh. ${ }^{41}$ Thus, the proportions of thrombus age vary, despite our study and these studies using the same widely accepted criteria for estimating thrombus age. ${ }^{27}$ Our study included patients who were treated more recently compared with those in previous studies, and all of our patients were treated using stent retrievers or large-bore aspiration catheters, which might provide higher reperfusion rates than previous devices. ${ }^{47}$ Thus, the proportion of thrombi that were resistant to recanalization therapy (i.e., older thrombi) may have been higher.

We confirmed that the interobserver agreement was moderate for the pathological estimation of thrombus age. However, to enhance the robustness of the analyses, we also considered CD163-positive cell density as another indicator of thrombus age. The results revealed that pathologically estimated thrombus age was correlated with the CD163positive tertiles and that the CD163 tertiles were associated with puncture-to-reperfusion time. Thus, regardless of whether age estimation was based on a pathological assessment or CD163-positive cell density, older thrombi appear to be associated with delayed reperfusion after mechanical thrombectomy for cerebral embolism.

\section{Race}

The proportions of stroke subtypes may differ according to each race. Interestingly, the proportion of intracranial stenosis is higher in Asians. ${ }^{48}$ Further, it was recently reported that clot strength is different between East Asians and Caucasians among coronary artery disease patients. ${ }^{49} \mathrm{Al}-$ though only embolic stroke patients were enrolled and the thrombi were mainly cardiogenic in this study, the generalizability of the results may be restricted by the study's design, as only Japanese patients were enrolled.

\section{Limitations}

The first limitation of this study is potential bias related to exclusion of patients whose thrombi could not be evaluated. Thus, it remains unclear whether our findings can be generalized to all patients who undergo mechanical thrombectomy for cerebral embolism. Second, we analyzed the thrombi according to the patient and not according to device pass, and we were only able to evaluate the most effective strategy for older thrombi (stent retriever and/or catheter aspiration) based on the rate of first-pass reperfusion. Third, we estimated thrombus features based on one section per each staining; thus, the evaluation may not accurately represent the entire sample. Finally, the retrieved thrombi might have contained "secondary thrombi," which form at the site of occlusion after embolism (therefore, they must be very new $)^{50}$; however, we did not attempt to distinguish between these two types and analyzed the entire section. Consequently, areas with intact granulocytes could be observed in most thrombi. Thus, we judged a thrombus as fresh only when intact granulocytes were dominantly observed thoroughly ( $>80 \%$ ) over a section, although no widely accepted cut-off value existed.

\section{Conclusion}

This study revealed that older thrombi were associated with longer times to reperfusion and more device passes, which resulted in poorer functional outcomes among patients with acute ischemic stroke. Further research is warranted regarding the mechanisms and pathophysiological roles of thrombus aging in embolisms. 


\section{What is known about this topic?}

- Thrombosis is a dynamic process, and a thrombus undergoes physical and biochemical changes.

- Thrombus age may influence the effectiveness of thrombolysis for venous thrombosis.

\section{What does this paper add?}

- An older thrombus delays reperfusion after mechanical thrombectomy for ischemic stroke.

- Older thrombi are associated with poorer functional outcomes.

- This effect of thrombus age was independent of erythrocyte content and the extent of NETosis.

\section{Funding}

This work was supported by JSPS KAKENHI [grants TK19K20729 and JSPS KAKENHI 21K15696].

\section{Conflict of Interest}

Dr. Kitano reports lecture fees from Stryker. Dr. Nakamura reports lecture fees from Johnson \& Johnson, Medtronic, and Stryker. Dr. Todo reports lecture fees from Medtronic. The other authors have no conflicts relevant to the contents of this article to disclose.

\section{Acknowledgment}

This study was supported by the Clinical Investigator's Research Project and Center for Medical Research and Education, Osaka University Graduate School of Medicine. The authors thank M. Kohara, T. Sawamura, M. Nihei, K. Nishiyama, M. Yoshida, and N. Iwachido for technical assistance with the specimen processing. We also thank M. Okamoto and M. Nishi for collecting the data.

\section{References}

1 Kamel H, Healey JS. Cardioembolic stroke. Circ Res 2017;120(03): 514-526

2 Furie B, Furie BC. Mechanisms of thrombus formation. N Engl J Med 2008;359(09):938-949

3 January CT, Wann LS, Calkins H, et al. 2019 AHA/ACC/HRS focused update of the 2014 AHA/ACC/HRS Guideline for the Management of Patients With Atrial Fibrillation: a report of the American College of Cardiology/American Heart Association Task Force on Clinical Practice Guidelines and the Heart Rhythm Society in Collaboration With the Society of Thoracic Surgeons. Circulation 2019;140(02):e125-e151

4 Nicklas JM, Gordon AE, Henke PK. Resolution of deep venous thrombosis: proposed immune paradigms. Int J Mol Sci 2020;21 (06):2080

5 Saha P, Humphries J, Modarai B, et al. Leukocytes and the natural history of deep vein thrombosis: current concepts and future directions. Arterioscler Thromb Vasc Biol 2011;31(03):506-512

6 Nosaka M, Ishida Y, Kimura A, Kondo T. Time-dependent organic changes of intravenous thrombi in stasis-induced deep vein thrombosis model and its application to thrombus age determination. Forensic Sci Int 2010;195(1-3):143-147
7 Helms CC, Ariëns RA, Uitte de Willige S, Standeven KF, Guthold M. $\alpha-\alpha$ Cross-links increase fibrin fiber elasticity and stiffness. Biophys J 2012;102(01):168-175

8 Collet J-P, Moen JL, Veklich YI, et al. The alphaC domains of fibrinogen affect the structure of the fibrin clot, its physical properties, and its susceptibility to fibrinolysis. Blood 2005;106 (12):3824-3830

9 Czaplicki C, Albadawi H, Partovi S, et al. Can thrombus age guide thrombolytic therapy? Cardiovasc Diagn Ther 2017;7(Suppl 3): S186-S196

10 Goyal M, Menon BK, van Zwam WH, et al; HERMES collaborators. Endovascular thrombectomy after large-vessel ischaemic stroke: a meta-analysis of individual patient data from five randomised trials. Lancet 2016;387(10029):1723-1731

11 Jansen IGH, Mulder MJHL, Goldhoorn RBMR CLEAN Registry investigators. Endovascular treatment for acute ischaemic stroke in routine clinical practice: prospective, observational cohort study (MR CLEAN Registry). BMJ 2018;360:k949

12 van den Berg LA, Dijkgraaf MG, Berkhemer OA, et al; MR CLEAN Investigators. Two-year outcome after endovascular treatment for acute ischemic stroke. N Engl J Med 2017;376(14):1341-1349

13 Khatri P, Yeatts SD, Mazighi M, et al; IMS III Trialists. Time to angiographic reperfusion and clinical outcome after acute ischaemic stroke: an analysis of data from the Interventional Management of Stroke (IMS III) phase 3 trial. Lancet Neurol 2014;13(06): 567-574

14 Alawieh A, Vargas J, Fargen KM, et al. Impact of procedure time on outcomes of thrombectomy for stroke. J Am Coll Cardiol 2019;73 (08):879-890

15 Hofmeister J, Bernava G, Rosi A, et al. Clot-based radiomics predict a mechanical thrombectomy strategy for successful recanalization in acute ischemic stroke. Stroke 2020;51(08):2488-2494

16 Bacigaluppi M, Semerano A, Gullotta GS, Strambo D. Insights from thrombi retrieved in stroke due to large vessel occlusion. J Cereb Blood Flow Metab 2019;39(08):1433-1451

17 Heo JH, Nam HS, Kim YD, et al. Pathophysiologic and therapeutic perspectives based on thrombus histology in stroke. J Stroke 2020;22(01):64-75

18 van Swieten JC, Koudstaal PJ, Visser MC, Schouten HJ, van Gijn J. Interobserver agreement for the assessment of handicap in stroke patients. Stroke 1988;19(05):604-607

19 Adams HP Jr, Bendixen BH, Kappelle LJ, et al. Classification of subtype of acute ischemic stroke. Definitions for use in a multicenter clinical trial. TOAST. Trial of Org 10172 in Acute Stroke Treatment. Stroke 1993;24(01):35-41

20 Barber PA, Demchuk AM, Zhang J, Buchan AM. Validity and reliability of a quantitative computed tomography score in predicting outcome of hyperacute stroke before thrombolytic therapy. ASPECTS Study Group. Alberta Stroke Programme Early CT Score. Lancet 2000;355(9216):1670-1674

21 Launes J, Ketonen L. Dense middle cerebral artery sign: an indicator of poor outcome in middle cerebral artery area infarction. J Neurol Neurosurg Psychiatry 1987;50(11):1550-1552

22 Schellinger PD, Chalela JA, Kang DW, Latour LL, Warach S. Diagnostic and prognostic value of early MR imaging vessel signs in hyperacute stroke patients imaged $<3$ hours and treated with recombinant tissue plasminogen activator. AJNR Am J Neuroradiol 2005;26(03):618-624

23 Liebeskind DS, Bracard S, Guillemin F, et al; HERMES Collaborators. eTICI reperfusion: defining success in endovascular stroke therapy. J Neurointerv Surg 2019;11(05):433-438

24 Schindelin J, Arganda-Carreras I, Frise E, et al. Fiji: an open-source platform for biological-image analysis. Nat Methods 2012;9(07): 676-682

25 Fuchs TA, Abed U, Goosmann C, et al. Novel cell death program leads to neutrophil extracellular traps. J Cell Biol 2007;176(02): 231-241 
26 Neeli I, Khan SN, Radic M. Histone deimination as a response to inflammatory stimuli in neutrophils. J Immunol 2008;180(03): 1895-1902

27 Rittersma SZ, van der Wal AC, Koch KT, et al. Plaque instability frequently occurs days or weeks before occlusive coronary thrombosis: a pathological thrombectomy study in primary percutaneous coronary intervention. Circulation 2005;111(09):1160-1165

28 Lau SK, Chu PG, Weiss LM. CD163: a specific marker of macrophages in paraffin-embedded tissue samples. Am J Clin Pathol 2004;122(05):794-801

29 Fabriek BO, Dijkstra CD, van den Berg TK. The macrophage scavenger receptor CD163. Immunobiology 2005;210(2-4):153-160

30 Furukoji E, Gi T, Yamashita A, et al. CD163 macrophage and erythrocyte contents in aspirated deep vein thrombus are associated with the time after onset: a pilot study. Thromb J 2016;14:46

31 Gi T, Kuroiwa Y, Yamashita A, et al. High signal intensity on diffusion-weighted images reflects acute phase of deep vein thrombus. Thromb Haemost 2020;120(10):1463-1473

32 Nosaka M, Ishida Y, Kimura A, Kondo T. Time-dependent appearance of intrathrombus neutrophils and macrophages in a stasisinduced deep vein thrombosis model and its application to thrombus age determination. Int J Legal Med 2009;123(03):235-240

33 McCaw ZR, Yin G, Wei L-J. Using the restricted mean survival time difference as an alternative to the hazard ratio for analyzing clinical cardiovascular studies. Circulation 2019;140(17):1366-1368

34 Royston P, Parmar MKB. The use of restricted mean survival time to estimate the treatment effect in randomized clinical trials when the proportional hazards assumption is in doubt. Stat Med 2011;30(19):2409-2421

35 Maekawa K, Shibata M, Nakajima H, et al. Erythrocyte-rich thrombus is associated with reduced number of maneuvers and procedure time in patients with acute ischemic stroke undergoing mechanical thrombectomy. Cerebrovasc Dis Extra 2018;8(01):39-49

36 Sporns PB, Hanning U, Schwindt W, et al. Ischemic stroke: histological thrombus composition and pre-interventional CT attenuation are associated with intervention time and rate of secondary embolism. Cerebrovasc Dis 2017;44(5-6):344-350

37 Yuki I, Kan I, Vinters HV, et al. The impact of thromboemboli histology on the performance of a mechanical thrombectomy device. AJNR Am J Neuroradiol 2012;33(04):643-648
38 Novotny J, Oberdieck P, Titova A, et al. Thrombus NET content is associated with clinical outcome in stroke and myocardial infarction. Neurology 2020;94(22):e2346-e2360

39 Longstaff C, Varjú I, Sótonyi P, et al. Mechanical stability and fibrinolytic resistance of clots containing fibrin, DNA, and histones. J Biol Chem 2013;288(10):6946-6956

40 Ducroux C, Di Meglio L, Loyau S, et al. Thrombus neutrophil extracellular traps content impair tPA-induced thrombolysis in acute ischemic stroke. Stroke 2018;49(03):754-757

41 Laridan E, Denorme F, Desender L, et al. Neutrophil extracellular traps in ischemic stroke thrombi. Ann Neurol 2017;82(02):223-232

42 Xie H, Kim K, Aglyamov SR, et al. Correspondence of ultrasound elasticity imaging to direct mechanical measurement in aging DVT in rats. Ultrasound Med Biol 2005;31(10):1351-1359

43 Lee YU, Lee AY, Humphrey JD, Rausch MK. Histological and biomechanical changes in a mouse model of venous thrombus remodeling. Biorheology 2015;52(03):235-245

44 Wong SL, Demers M, Martinod K, et al. Diabetes primes neutrophils to undergo NETosis, which impairs wound healing. Nat Med 2015;21(07):815-819

45 Porrello A, Leslie PL, Harrison EB, et al. Factor XIIIA-expressing inflammatory monocytes promote lung squamous cancer through fibrin cross-linking. Nat Commun 2018;9(01):1988

46 Niesten JM, van der Schaaf IC, van Dam L, et al. Histopathologic composition of cerebral thrombi of acute stroke patients is correlated with stroke subtype and thrombus attenuation. PLoS One 2014;9(02):e88882

47 Broussalis E, Trinka E, Hitzl W, Wallner A, Chroust V, KillerOberpfalzer M. Comparison of stent-retriever devices versus the Merci retriever for endovascular treatment of acute stroke. AJNR Am J Neuroradiol 2013;34(02):366-372

48 Kim BJ, Kim JS. Ischemic stroke subtype classification: an Asian viewpoint. J Stroke 2014;16(01):8-17

49 Jeong YH, Kevin B, Ahn JH, et al. Viscoelastic properties of clot formation and their clinical impact in East Asian versus Caucasian patients with stable coronary artery disease: a COMPARE-RACE analysis. J Thromb Thrombolysis 2021;51(02):454-465

50 Qazi EM, Sohn SI, Mishra S, et al. Thrombus characteristics are related to collaterals and angioarchitecture in acute stroke. Can J Neurol Sci 2015;42(06):381-388 